\title{
I year follow up implantable collamer lenses (ICLs) for the treatment of pediatric anisometropic amblyopia
}

\begin{abstract}
Purpose: To evaluate the safety and efficacy during at least 1-year follow-up of visian implantable collamer lens ICL to correct high anisometropia in amblyopic children who were non-compliant with spectacles or contact lenses.

Methods: A prospective non comparative interventional case series study of 12 eyes of 12 children with high anisometropia who underwent ICL implantation (phakic posterior chamber IOL). Patient age at the time of implantation ranged from 2 to 15 years. Mean preoperative spherical equivalent refraction was -10 diopters (D). Mean $\operatorname{logMAR}$ uncorrected visual acuity (UCVA) was .03 and corrected distance visual acuity (CDVA) was .3 occlusion therapy was done after surgery in all cases and follow up for a period 1-2 years was done in all cases. Cases with less than 1 year follow up were excluded from the study.

Results: UCVA and CDVA improved in all children. At 12 months, mean decimal UCVA and CDVA were 0.6 and 0.8 respectively (P value less than .01). No loss of CDVA was detected in any patient. Endothelial cell loss was comparable to normal age related changes in endothelial cell count.

Conclusion: ICL provided significant improvement in visual acuity and spherical equivalent, which suggests that it may be effective, safe and predictable for correcting pediatric refractive errors producing anisometropic amblyopia.
\end{abstract}

Keywords: ICL, childhood, pediatric, anisometropia, amblyopia, phakic iol, refractive surgery
Volume 7 Issue 5 - 2017

\author{
Ahmed Elmassry, Amr Ahmed Said, \\ Mohammed Ahmed Elmasry \\ Alexandria University, Egypt
}

Correspondence: Ahmed Elmassry, Moustafa Kamel Street, Alexandria, Egypt, Tel 002-012-2152435,

Email ahmad.elmassry@gmail.com

Received: September 23, 2017 | Published: October 17, 2017

\section{Introduction}

Population-based studies attempt to determine the prevalence of untreated refractive error and its disability have estimated 5-7\% of preschool children to have significant refractive errors and $1-4 \%$ has amblyopia. ${ }^{1}$ Most children with refractive errors can be treated effectively and safely with spectacles or contact lenses., ${ }^{2,3}$ In most cases of refractive amblyopia, conventional therapy is effective. ${ }^{4}$ Conventional amblyopia therapy consists of the following steps: clearing the ocular media if there is a visual obstruction such as a leukoma, visually significant cataract, or vitreous hemorrhage; correcting significant refractive error with either spectacles or contact lenses; and occlusion or pharmacologic and/or optical penalization of the fellow eye. ${ }^{5,6}$ However, these traditional forms of treatment have proven inadequate in a subset of children, in particular those with high-magnitude of ametropia who are spectacle non compliant as well as children with neurobehavioral abnormalities related to autism, cerebral palsy, craniofacial anomalies, ear deformities, or neck hypotonia that preclude the use of spectacle correction. ${ }^{7,8}$

Witnessing the impressive effect of modern refractive surgery in adults, many ophthalmologists started investigating modern refractive surgery in the pediatric population. The first report of laser-assisted refractive surgery in the pediatric population was published in $1995 .{ }^{9}$ Since then, many small case series have been published reporting mild to moderate improvements in vision and stable refractive results in the short-term and intermediate-term follow-up. ${ }^{10,11}$ Pediatric refractive surgery indications include children under the age of 7 with anisometropic amblyopia who are intolerant of spectacles or contact lenses. The categories of pediatric patients that may be considered for refractive surgery is: Neuro developmentally abnormal patients for whom traditional therapy has failed and neuro developmentally normal patients with a high-risk of developing dense amblyopia: high refractive errors or significant anisometropia belong to the second group. Refractive surgery may have better outcomes than spectacles or contact lenses in this group. This may be because of the dramatic decrease in the amount of anisometropia after refractive surgery. This, in turn, often leads to better postoperative uncorrected visual acuity. Finally, because of the decreased spherical equivalent, such patients may be more compliant with glasses or contact lens wear after refractive surgery. ${ }^{10-12}$

Two main Controversies about refractive surgery exist even among those who advocate such procedures the age at which to perform the refractive surgery and choice of technique used. ${ }^{13}$ Some authors recommend treating children during the neuroplastic years, when the possibility of reversing the amblyopia is greatest. Conversely, issues of refractive instability and more aggressive immune response in younger children may favor older age for treatment. Further, younger children may not be able to comply with the procedure, which 
necessitates the need for general anesthesia with its inherent risks and its effect on optical centration. Finally, it is unclear whether the adult nomograms are accurate when applied to the very young patient. ${ }^{14}$

The second controversy among pediatric refractive surgeons is about the technique used to treat the refractive error as corneal anatomy differs in children from that of adults. Each technique has advantages and drawbacks. Laser assisted in situ keratomelesis (LASIK) advocates argue that photorefractive keratectomy (PRK), especially in higher myopes, is associated with myopic regression and corneal haze (higher rate in children due to more aggressive healing response and failure to comply to long-term steroid therapy). ${ }^{15,16}$ PRK may necessitate the use of topical corticosteroids for several months, with the risk of elevated intraocular pressure and possible glaucoma as well as cataract formation. However, proponents for PRK argue that PRK has a better risk profile than LASIK. ${ }^{17}$ For anisometropia or severe ametropia +6 to $-12 \mathrm{D}$, LASIK procedure can be used. Femto second Laser for flap making can help in making flaps with better healing and precise dimensions. ${ }^{18}$ Until these procedures are compared in a large, multicenter, randomized trial, the use of LASIK, or PRK is left to the expertise of the treating physician and the specific patient case. Children are more likely to rub their eyes than adults, with a theoretically increased risk of flap dislocation or loss. PRK also reduces or avoids other LASIK-related complications, including flap tear or striae and keratectasia. ${ }^{16,17}$

Regression is seldom due to increase in the axial length. After age 2-3 years, axial length changes tend to be minor in highly ametropicphakic pediatric eyes. The high rate of regression is attributable to the enhanced healing properties in young eyes, such as keratocyte-mediated regrowth and epithelial hyperplasia. Average regression can be around 1 diopter/year. ${ }^{19,20}$ Clear lens extraction/ refractive lens exchange for children with ametropia exceeding $20 \mathrm{D}$ (the upper limit for phakic IOL power) or anterior chamber depth less than $3.2 \mathrm{~mm}$. Standard pediatric lensectomy, posterior capsulectomy and anterior vitrectomy techniques are employed, using a high-speed 23-gauge vitrector and separate anterior chamber maintainer. If needed to achieve emmetropia, a foldable, acrylic IOL (monofocal or multifocal) is implanted, depending on axial length and lens power calculations. A primary capsulectomy/anterior vitrectomyis advisable because of the high rate of formation of dense, posterior capsule fibrosis in these pediatric eyes when the capsule is preserved. Removing the lens abolishes the ability to accommodate. The peripheral retina should be examined in detail by depression. However high incidence of post operative retinal detachment makes this procedure almost obsoletre in the developed countries. ${ }^{21-23}$ Phakic IOL implantation is not subject to significant regression and may be considered the preferred method currently for surgical correction of pediatric myopia and hyperopia beyond the range of laser vision correction. ${ }^{24}$

The myopic (Verisyse) lens in the USA is supplied by Advanced Medical Optics (Santa Ana, California, USA) Safe insertion, and lower long-term risk for loss of corneal endothelial cells, requires an anterior chamber depth $3.2 \mathrm{~mm}$ or greater. A small iridotomy or iridectomy is performed during the procedure to reduce the chance of pupillary block. Because children's eyes heal rapidly, a superior clear cornea incision can be employed, which achieves the therapeutic effect of a relaxing limbal incision. Common with-the-rule, preoperative astigmatism is reduced by about $50 \%$. Because these implants lie immediately adjacent to the iris pigment layer and lens, they pose greater risk for pigment dispersion and cataract formation in a pediatric eye. ${ }^{24,25}$ The current model, the Visian ICL V4C, is a rectangular single-piece IOL, 7.5 to $8.0 \mathrm{~mm}$ wide, available in 4 overall lengths: 11.5 to $13.0 \mathrm{~mm}$ in $0.5 \mathrm{~mm}$ steps for myopic correction and 11.0 to $12.5 \mathrm{mmin} 0.5 \mathrm{~mm}$ steps for hyperopic correction. The optic diameter ranges from 4.65 to $5.5 \mathrm{~mm}$ in myopic ICLs, depending on the dioptric power. It is always $5.5 \mathrm{~mm}$ in hyperopic ICLs. The available power ranges from -3.0 to $-23.0 \mathrm{D}$ for myopic IOLs, from +3.0 to $+22.0 \mathrm{D}$ for hyperopic ICLs, and with an added positive cylinder of 1.0 to $6.0 \mathrm{D}$ for toric ICLs. ${ }^{23,26}$

The ICL can be inserted through a $3.0 \mathrm{~mm}$ incision using a microinjector. It has orientation markings on its haptics, allowing control during the unfolding maneuver. The thickness is less than $50 \mathrm{~mm}$ in the optic zone, 500 to $600 \mathrm{~mm}$ in the hapticmzone, and $100 \mathrm{~mm}$ in the haptic footplates, which are theoretically positioned in the cilliary sulcus using a spatula specially designed for the ICL. The basic design change of the ICL V4 addresses the vaulting. This model has an additional 0.13 to $0.21 \mathrm{~mm}$ anterior vault due to the steeper radius of curvature of the base curve, which depends on the dioptric power. The higher vault provides a greater space between the posterior surface of the ICL and the anterior surface of the crystalline lens, which allows fluid change of nutrients and prevents contact between the ICL and the crystalline lens. ${ }^{26,27}$

\section{Patients and methods}

A prospective analysis of 12 eyes (anisometropic and amblyopic, noncompliant to traditional treatment) with at least one year followup. Implantation of STAAR Visian ICL. Mean age of the patients was 8 years (range 2-15years).

\section{Inclusion criteria}

Children with high anisometropicmyopic amblyopia with endothelial cell count (ECC) of at least $2000 \mathrm{cells} / \mathrm{mm}^{2}$ and a central anterior chamber depth (ACD) of at least $3.0 \mathrm{~mm}$.

\section{ICL Selection}

The ICL overall diameter depends on the ciliary sulcus diameter and should provide perfect stability with no excess of compression forces to the sulcus and allow correct vaulting. Excessive vaulting (more than 750 microns) due to an ICL that is too long may cause angle-closure, papillary block glaucoma, or pigmentary dispersion glaucoma. Insufficient vaulting (less than 250 microns) due to an ICL that is too short increases the risk for cataractogenesis due to the contact between the posterior surface of the ICL and the anterior surface of the crystalline lens. The internal diameter of the ciliary sulcus Is approximated and is depended on a white to white (WTW) measurement. The ICL's diameter is oversized 0.5 to $1.0 \mathrm{~mm}$ from the WTW measurement in myopic eyes

\section{Surgical technique}

Correct loading of the ICL in the cartridge and the injector is essential for correct and easy implantation. Using a modified McPherson forceps with long, blunt, curved tips, the ICL is grasped and checked under the operating microscope. The ICL has 2 tiny holes on the footplates (distal right and proximal left) that allow correct anterior-posterior orientation. The cartridge is filled with OVD. The ICL is loaded with dome up, being especially careful of the haptic positions to avoid rupturing them. A piece of soft material, the Star foam tip, is positioned to protect the ICL from contact with the plunger of the shooter. 
Broad pharmacological mydriasis is essential for uneventful implantation. The ICL can be inserted through a sub-3.0 $\mathrm{mm}$ incision. 2 paracenteses incisions are made to enable easier implantation of the haptics in the ciliary sulcus. The anterior chamber is filled with a cohesive OVD to protect the corneal endothelium and crystalline lens from surgical trauma. The cartridge is inserted bevel down, and the ICL is carefully injected. Finally, the haptics are gently pushed under the iris with a blunt spatula. While centration of the ICL and position of the haptics in the ciliary sulcus are checked, complete extraction of the OVD is mandatory to prevent postoperative ocular hypertension. Finally, the wound is hydrated.

\section{Postoperative treatment and follow up}

The topical antibiotic moxifloxacin (Vigamox, Alcon Laboratories, USA), steroids (prednisolone; Pred Forte, Allergan, Ireland) were used postoperatively every hour during the first day, and then every 4 hours for 1 month. The antibiotic dose timing was every hour for the first day, which then decreased to every 4 hours for 10 days. Patients/parents were instructed to avoid eye rubbing. All patients were examined at day 1 for ICL position, centration and vaulting. Complete ophthalmological test, including UCVA, BCVA, manifest and cycloplegic refraction and specular microscopy is carried out on 1 , 3 and 6 months, postoperatively whenever possible. All complications, if any, were documented. Another follow up visit at completion of $1^{\text {st }}$ and $2^{\text {nd }}$ year after surgery. Cases with less than 1 year follow up were excluded from the study.

\section{Statistical analysis}

Data were analyzed using SPSS Software (Statistics version 23 for Windows, USA). Quantitative data were described using minimum

Table I Summary of preoperative and postoperative outcomes

\begin{tabular}{|c|c|c|c|c|}
\hline SD \pm Mean & Preoperative & $\begin{array}{l}\text { Postoperative } \\
\text { I month }\end{array}$ & $\begin{array}{l}\text { Postoperative } \\
6 \text { months }\end{array}$ & $\begin{array}{l}\text { Postoperative } \\
\text { I year }\end{array}$ \\
\hline $\begin{array}{l}\text { UCVA } \\
\text { (Decimal) }\end{array}$ & $0.12(20 / 200) \pm 0.21(20 / 100)$ & $\begin{array}{l}0.63(20 / 30) \pm 0.32 \\
(P<0.00 I)\end{array}$ & $\begin{array}{l}0.6(20 / 30) \pm 0.29 \\
(P<0.001)\end{array}$ & $\begin{array}{l}0.53(20 / 40) \pm 0.26 \\
(P<0.00 I)\end{array}$ \\
\hline BCVA & $0.48 \pm 0.20$ & $0.86(20 / 25) \pm 0.37(\mathrm{P}<0.00 \mathrm{I})$ & $\begin{array}{l}0.8 \mathrm{I}(20 / 25) \pm 0.22 \\
(P<0.00 \mathrm{I})\end{array}$ & $\begin{array}{l}0.86(20 / 25) \pm 0.37 \\
(P<0.00 I)\end{array}$ \\
\hline $\begin{array}{l}\text { Spherical } \\
\text { Equivalent }\end{array}$ & $-11.28 \pm 4.70$ & $\begin{array}{l}-1.28 \pm 2.12 \\
(P<0.001)\end{array}$ & $\begin{array}{l}-I .34 \pm 2.82 \\
(P<0.00 I)\end{array}$ & $\begin{array}{l}-1.82 \pm 2.90 \\
(P<=0.001)\end{array}$ \\
\hline ECC & $\begin{array}{l}2599.34+549.92 \text { cells } / \mathrm{mm}^{2} \\
(P=0.434)\end{array}$ & $\begin{array}{l}2517.34 \_411.92 \text { cells } / \mathrm{mm}^{2} \\
(P=0.234)\end{array}$ & $\begin{array}{l}2531.34 \_399.92 \text { cells } / \mathrm{mm}^{2} \\
(P=0.654)\end{array}$ & $\begin{array}{l}2499.34 \_449.92 \text { cells } / \mathrm{mm}^{2} \\
(P=0.455)\end{array}$ \\
\hline
\end{tabular}

Abbreviations: UCVA, uncorrected visual acuity; BCVA, best corrected visual acuity; ECC, endothelial cell count

\section{Intraoperative and postoperative complications}

No intraoperative complication was reported we have not encountered cases of post operative cataract nor papillary block glaucoma. All children had retinal examination at 1 year follow up. No a single case of retinal detachment, giant retinal break nor a macular hole was seen. Endothelial cell count was evaluated during follow up by specular microscope for children at age capable of sitting for specular microscopy to capture corneal endothelial image. During follow up period no significant loss of it was documented denoting safety of ICL for children endothelium and maximum, as well as mean and standard deviation. Comparison between different periods was assessed using an ANOVA test with repeated measures and Bonferroni correction. Significance test results were quoted as two-tailed probabilities. Significance of the obtained results was determined at the $5 \%$ level.

\section{Results}

During the study period, the ICL s was implanted more than 20 eyes of children with anisometropic amblyopia refractory or non compliant to traditional treatment options. Only cases ICL implantation that completed at least 1 year of follow-up was included in the study. This study included 12 eyes of 12 patients. The patient age ranged between 2 and 15 years (mean \pm SD; $8 \pm 3$ ). There were 8 boys and 4 girls.

\section{Visual outcomes}

Table 1 summarizes the preoperative and postoperative outcomes. The UCVA was significantly better $(\mathrm{P}<0.001)$ than preoperative measurements at all follow-up visits. At 1 year, the mean decimal UCVA improved from $0.12(20 / 200) \pm 0.21$ preoperatively to $0.73(20 / 28) \pm 0.32$.Comparison of UCVA at different follow-up periods did not show any statistically significant difference. The BCVA was $0.5(20 / 40)$ or better in 6 out of 12 eyes (50\%) at 1 month, in 8 out of 12 eyes at 6 months, and in 9 out of 12 eyes (75\%) at 1 year. The improvement in BCVA increased from $0.48(20 / 50) \pm 0.20$ preoperatively to $0.86(20 / 25) \pm 0.37$ after 1 year postoperatively, and $75 \%$ of eyes showed improvement in 2 lines or more. No eye of our series lost BCVA lines. In all patients, marked improvement in spherical equivalent was observed.Mean spherical equivalent refractions were significantly lower $(\mathrm{P}<0.001)$ at 1 year months, a reduction from- $11.28 \pm 4.70$ to $-1.02 \pm 2.9$. 
parents reported an improvement in their child's quality of life. A gain of 3 or more Snellen lines, as well as recovery of binocular vision was observed in two patients. Another follow-up study by Lesueur $\&$ Arne $^{29}$ reported outcomes of same type -phakic IOL to correct high myopia and amblyopia in 12 eyes of children aged 3-16 years old. These children had a mean preoperative spherical equivalent of $-12.7 \mathrm{D}$ and were followed up for a period of 20.5 months. Six patients recovered binocular vision and showed improvement in QOL. Also there was no report of any complications. Alio et $a .^{30}$ report the longest follow-up period of 5 years, following PC-pIOL implantation in a child with high anisometropic amblyopia. Improvement in visual acuity of one logMAR line was reported, and no complications were seen (Figure 1\&2).
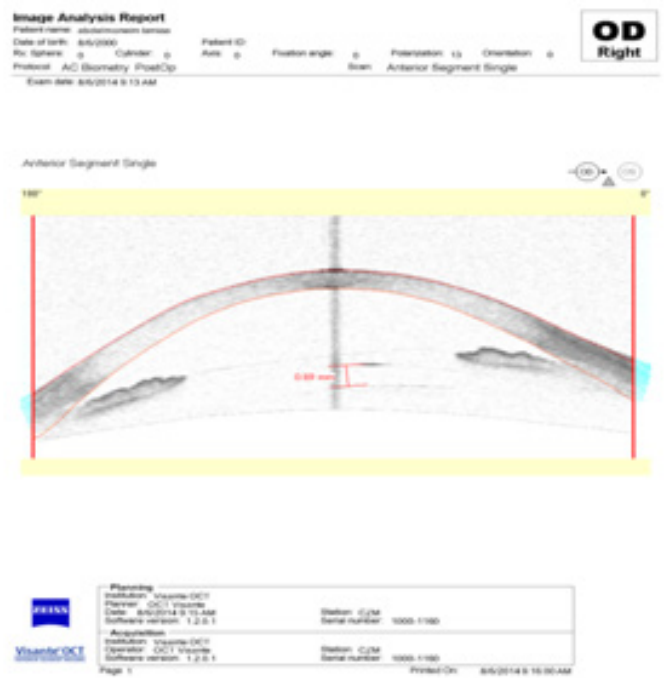

Figure I Anterior segment OCT I year following surgery showing ICL in place.

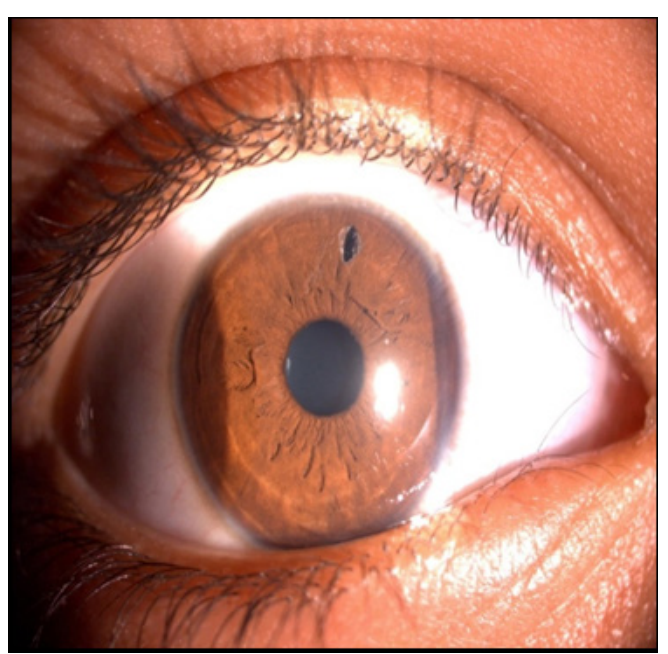

Figure 2 Slit lamp examination of case number 4 one year after surgery

Reports of complications in the pediatric population are rare; however, this is likely due to the low overall numbers of PC-pIOLs in children. Reports of adverse events in the pediatric refractive surgeries are rare due to the limited number of surgeries performed; however, complications in adults include cataract formation, uveitis, IOL dislocation, pigment dispersion, and endothelial cell loss.
The most significant concern in the pediatrics is long-term corneal endothelial cell loss. This is particularly important due to long life expectancy of these children, as well as potential for eye rubbing. However, experience in the pediatric population to date is limited and this is compounded by difficulties in obtaining accurate endothelial cell counts in children. In the studies of Pirouzian Alio et al., ${ }^{31}$ the endothelial cell loss rate varied over the course of 3-5 years between $6.5 \%$ and $15.2 \%$ in different phakic IOLs in children. Our study has some potential limitations-the lack of a comparative group (use of spectacle/contact lenses only at the same follow up period), the absence of long-term follow-up data about safety and refractive stability, the lack of data about changes in higher-order aberrations and need and success of enhancement procedures later in life.

\section{Conclusion}

ICL provided significant improvement in visual acuity and spherical equivalent, which suggests that it may be effective, safe and predictable for correcting pediatric refractive errors producing anisometropic amblyopia.

\section{Conflicts of interest}

The authors have no financial or proprietary interest in any materials or methods presented herein.

\section{References}

1. Afsari S, Rose KA, Gole GA, et al. Prevalence of anisometropia and its association with refractive error and amblyopia in preschool children. $\mathrm{Br}$ J Ophthalmol. 2013; 97(9):1095-1099.

2. Marsh-Tootle WL, Dong LM, et al. Myopia progression in children wearing spectacles Vs. switching to contact lenses. Optom Vis Sci. 2009;86(6):741-747.

3. Schlote T, Kriegerowski M, Bende T, et al. Mesopic vision in myopia corrected by photorefractive keratectomy, soft contact lenses, and spectacles. J Cataract Refract Surg. 1997;23(5):718-725.

4. Verma A, Singh D. Active vision therapy for pseudophakic amblyopia. $J$ Cataract Refract Surg. 1997;23(7):1089-1094.

5. Li T, Shotton K. Cochrane review: conventional occlusion versus pharmacologic penalization for amblyopia. Evi Based Child Health 2010;5(4):1873-1909.

6. Potts AM, Nagaya T. Studies On The Visual Evoked Response: III. Strabismus amblyopia and hysterical amblyopia. Documenta Ophthalmologica. 1969;26(1):394-402.

7. Robaei D, Kifley A, Rose KA, et al. Refractive error and patterns of spectacle use in 12-year-old Australian children. Ophthalmology. 2006;113(9):1567-1573.

8. Robaei D, Kifley A, Rose KA, et al. Correctable and non-correctable visual impairment in a population-based sample of 12-year-old Australian children. Ophthalmology. 2006;113(9):1567-1573.

9. Singh D. Photorefractive keratectomy in pediatric patients. J Cataract Refract Surg. 1995;21(6):630-632.

10. Tychsen L, Hoekel J. Refractive surgery for high bilateral myopia in children with neurobehavioral disorders: 2 . Laser-Assisted subepithelial keratectomy (LASEK). J AAPOS. 2006;10(4):364-370.

11. Tychsen L, Packwood E, Berdy G. Correction of large amblyopiogenic refractive errors in children using the excimer laser. $J$ AAPOS. 2005;9(3): $224-233$. 
12. Saeed AM, Abdrabbo MA. LASIK As An Alternative Line To Treat Noncompliant Esotropic Children. Clin Ophthalmol. 2011;1795-1801.

13. Gurwood AS. LASIK Surgery in Children. Optometry. Journal of the American Optometric Association. 2004;75(5): 276.

14. Kohnen T. Refractive Surgery in Children. Journal of Cataract \& Refractive Surgery. 2007;33(12):2001.

15. Kuo IC, Lee SM, Hwang DG. Late-Onset Corneal Haze And Myopic Regression After Photorefractive Keratectomy (PRK). Cornea. 2004;23(4):350-355.

16. Katlun T, Wiegand W. Haze Und Regression Nach Photo refractiver Keratektomie (PRK). Der Ophthalmologe. 2000;97(7):487-490.

17. Schipper I. PRK, LASIK, LASEK Und Epi-LASIK: WelcheIst Die Beste Methode? Klinische Monatsblätterfür Augenheilkunde. 2007; pp. 224 R_291.

18. Pietilä J, Huhtala A, Mäkinen P,et al. Flap characteristics, predictability, and safety of the ziemer FEMTO LDV femtosecond laser with the disposable suction ring for LASIK. Eye. 2013;28(1):66-71.

19. Takahashi A, Ito Y, Iguchi Y, Yasuma TR, et al. Axial length increases and related changes in highly myopic normal eyes with myopic complications in fellow eyes. Retina. 2012;32(1):127-133.

20. Rapuano CJ. Live or Let Die: Epithelial Flap Vitality and Keratocyte Proliferation Following LASEK And Epi-LASIK In Human Donor And Porcine Eyes. Yearbook of Ophthalmology. 2012; pp. 53-54.

21. Ripandelli G, Billi B, Fedeli R, et al. Retinal detachment after clear lens extraction in 41 eyes with high axial myopia. Retina. 1996;16(1): 3-6.

22. King JS, Priester WB, Netland PA. Pseudophakic glaucoma and vision loss after clear lens extraction for high myopia. Annals of Ophthalmology. 2004;36(1): 53-54.
23. Emarah AM, El-Helw MA, Yassin HM. Comparison of clear lens extraction and collamer lens implantation in high myopia. Clin Ophthalmol. 2010;4: 447-454.

24. Rosman M, Alió JL, Ortiz D, Pérez-Santonja. Refractive Stability of LASIK with the VISX 20/20 Excimer Laser Vs ZB5M Phakic IOL Implantation in Patients with High Myopia (>-10.00 D): A 10-Year Retrospective Study. Journal of Refractive Surgery. 2011;27(4): 279-286.

25. Pirouzian A, Bansal P, O’Halloran H. Phakic IOL In Children. Ophthalmology. 2007;114(1):194-195.

26. Bhandari V, Karandikar S, Reddy JK, et al. Implantable collamer lens v4b and v4c for correction of high myopia. J Curr Ophthalmol . 2016;27(3-4): 76-81.

27. Ganesh S, Brar S. Comparison of Surgical Time and IOP Spikes with two Ophthalmic Viscosurgical Devices Following Visian STAAR (ICL, V4c Model) Insertion in the Immediate Postoperative Period. Clin Ophthalmol. 2016;207-211.

28. Lesueur LC, Arne JL. Phakic posterior chamber lens intraocular implantation in children with high myopia. J Cataract Refract Surg. 1999;25(12):1571-1575.

29. Lesueur LC, Arne JL. Phakic intraocular lens to correct high myopia amblyopia in children. J Refract Surg. 2002;18(5):519-523.

30. Alio JL, Toffaha BT, Laria C, et al. Phakic intraocular lens implantation for treatment of anisometropia and amblyopia in children: 5-year followup. J Refract Surg. 2011;27(7):494-501.

31. Pirouzian A, Ip KC. Anterior chamber phakic intraocular lens implantation in children to treat severe anisometropic myopia and amblyopia: 3-year clinical results. J Cataract Refract Surg. 2010;36(9):1486-1493. 